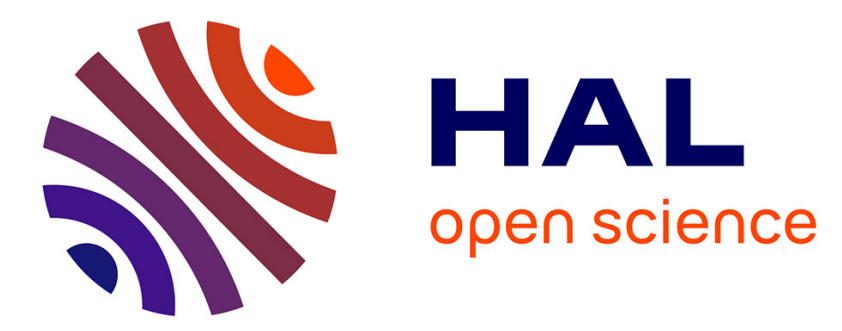

\title{
Experimental determination of the energy optimum for the transport of floating particles in pipes
}

Denis Edelin, Pierre-Clément Czujko, Cathy Castelain, Christophe Josset, Francine Fayolle

\section{- To cite this version:}

Denis Edelin, Pierre-Clément Czujko, Cathy Castelain, Christophe Josset, Francine Fayolle. Experimental determination of the energy optimum for the transport of floating particles in pipes. Experimental Thermal and Fluid Science, 2015, 68, pp.634-643. 10.1016/j.expthermflusci.2015.06.018 . hal-01974865

\section{HAL Id: hal-01974865 \\ https://hal.science/hal-01974865}

Submitted on 19 Nov 2019

HAL is a multi-disciplinary open access archive for the deposit and dissemination of scientific research documents, whether they are published or not. The documents may come from teaching and research institutions in France or abroad, or from public or private research centers.
L'archive ouverte pluridisciplinaire HAL, est destinée au dépôt et à la diffusion de documents scientifiques de niveau recherche, publiés ou non, émanant des établissements d'enseignement et de recherche français ou étrangers, des laboratoires publics ou privés. 


\title{
Experimental determination of the energy optimum for the transport of floating particles in pipes
}

\author{
Denis Edelin $^{\mathrm{a}, \mathrm{b}}$, Pierre-Clement Czujko ${ }^{\mathrm{a}, \mathrm{c}}$, Cathy Castelain ${ }^{\mathrm{a}, *}$, Christophe Josset ${ }^{\mathrm{a}}$, Francine Fayolle ${ }^{\mathrm{b}}$ \\ a Laboratoire de Thermocinetique CNRS UMR 6607, Polytech Nantes, 44306 Nantes, France \\ ${ }^{\mathrm{b}}$ GEPEA - CNRS UMR 6144, ONIRIS, 44322 Nantes, France \\ ${ }^{\text {c } C E T H I L ~-~ C N R S ~ U M R ~ 5008, ~ I N S A ~ d e ~ L y o n, ~} 69621$ Villeurbanne, France
}

\section{A R T I C L E I N F O}

\section{Article history:}

Received 16 September 2014

Received in revised form 25 June 2015

Accepted 25 June 2015

Available online 11 July 2015

\section{Keywords:}

Solid-liquid flow

Floating particles

Slurries

Pressure drop

Transport efficiency

\begin{abstract}
A B S T R A C T
This paper reports the results of an experimental investigation into the transport of fluids composed of water and polypropylene particles, in order to study the transport of floating particles.

The determination of the pressure drop and the delivered concentration, for a wide combination of flow rates $\left(0-0.5 \mathrm{~kg} \mathrm{~s}^{-1}\right)$, solid loads $(0-25 \% \mathrm{vol})$ and particles mean sizes $(341-756 \mu \mathrm{m})$, enabled the transport efficiency for this kind of slurry in a circular horizontal pipe ( $30 \mathrm{~mm}$ diameter) to be measured.

The results are displayed in relation to flow patterns; they show that the size of the particles has a little impact on the transport efficiency. On the contrary, the flow rate and the volumetric concentration have a strong impact on efficiency, because of their large contribution to the flow pattern. The best operating conditions are obtained close to the limit deposition velocity, when the fully suspended pattern is reached. The solids are then well transported with no waste of energy due to a too high velocity. The optimum concentrations, for a wide range of high efficiency are around $20-25 \%$ vol.
\end{abstract}

(c) 2015 Elsevier Inc. All rights reserved.

\section{Introduction}

Biphasic liquid-solid mixtures are generally known slurry; if the solid particles are dispersed in the liquid, the term used is a suspension. However, apart from colloidal suspensions, the apparent weight of the particles plays an important role in the suspensions transport. The apparent weight entails particles stratification. Most slurries are sedimentary, i.e. the particles are heavier than the liquid. In the case of an ice slurry, the phenomenon is inverted. This kind of slurry is much less observed in nature or in industrial applications.

Slurries suspensions transported through pipe-lines are essential for different applications and have drawn considerable attention in recent years in mining and mineral processing [1], ice slurry transport [2], pipeline transport of agricultural slurries in chemical plants, for example wheat straw and corn stover [3], sand removal [4], dredging and tailing disposal [5]. In many cases, the transported slurries consist of small size particles (usually up to a few tens of microns). However, coal-water mixtures, and sand

\footnotetext{
* Corresponding author.

E-mail addresses: denis.edelin@univ-nantes.fr (D. Edelin), pierre-clement. czujko@insa-lyon.fr (P.-C. Czujko), cathy.castelain@univ-nantes.fr (C. Castelain), christophe.josset@univ-nantes.fr (C. Josset), francine.fayolle@oniris-nantes.fr (F. Fayolle).
}

or gravel slurries can contain much coarser particles (up to a few millimeters).

In the context of the increasingly urgent need to reduce carbon emissions, it is highly desirable to investigate options to reduce power consumption in handling slurry in mining and mineral operations, and in slurry transport in general. Similar to slurry mixing tanks, slurry transport in pipelines is an established technology in the mining and mineral processing industry. There is a wealth of literature on this topic, dating back to the 1960s. Most researchers [6-8] in slurry pipeline transport are concerned with predicting pressure loss, or determining the minimum conveying velocity (deposition velocities) for safe transport and on the impact of various parameters including solids loading, particle shape and rheology on pressure gradient, and on methods to predict conveying characteristics accurately.

To a large extent, the pressure gradient data reported in most literature [7] have been obtained to determine the power consumption required when selecting and sizing slurry pumps, and to optimize the pipeline design parameters (e.g. pipe diameter, percentage of fine particles) so as to minimize the capital cost and energy consumption. Pullum and McCarthy [8] compiled Specific Energy Consumption (SEC) data from a total of 20 publications, and concluded that high concentration coarse particles mixed in non-Newtonian viscous carrier fluids (such as fly ash), where the solids concentration is typically more than $40 \%$ by 


$\begin{array}{ll}\text { Nomenclature } \\ m & \text { mass }[\mathrm{kg}] \\ \dot{\mathrm{m}} & \text { mass flow rate }[\mathrm{kg} / \mathrm{s}] \\ V & \text { volume }\left[\mathrm{m}^{3}\right] \\ \dot{\mathrm{V}} & \text { volume flow rate }\left[\mathrm{m}^{3} / \mathrm{s}\right] \\ D & \text { pipe diameter }[\mathrm{m}] \\ L & \text { pipe length }[\mathrm{m}] \\ d & \text { particle size }[\mathrm{m}] \\ g & \text { standard gravity }\left[\mathrm{m} / \mathrm{s}^{2}\right] \\ \rho & \text { density }\left[\mathrm{kg} / \mathrm{m}^{3}\right] \\ \mu & \text { dynamic viscosity }[\mathrm{Pa} \mathrm{s}] \\ \tau_{\mathrm{w}} & \text { wall shear stress }[\mathrm{Pa}] \\ \Delta \mathrm{P} & \text { pressure differential }[\mathrm{Pa}]\end{array}$

volume, can be hydraulically conveyed at low velocities with a SEC comparable to or even lower than that required for conventional dilute solids phase systems. These particles can have a high density (such as iron, or sand) compared to the main fluid and are considered settling slurries. On the contrary, others particles can have a low density (ice slurry for example).

Some studies have investigated the dependence of the diameter and the lifetime of pipelines transporting solid-liquid mixtures on the solid particle size. In order to achieve these goals, a life-cycle cost analysis model has been developed in which the particle size effects are incorporated by the weighted mean diameter [9].

Slurry flow behavior, can be analyzed by the equilibrium of forces, that are present and that control particles displacement. In the liquid-solid biphasic mixing case, there are numerous interactions. The simplification of the unperturbed flow by the presence of particles is applicable to very dilute suspensions, because the local perturbation of the flow by a particle does not reach others particles. However, in the case of undiluted particles flows, the particles interact with each other. It is then necessary to determine the long-range and short-range hydrodynamics interactions between them. The large number of these forces make it unrealistic to try to develop numerical resolutions, that take into account the complete physical model. There are some numerical studies but for a small number of particles, or for small volumes by neglecting the number effects, and to obtain the macroscopic and homogenization properties of the solutions [10].

In practice, for slurry applications, the overall behavior of the mixture is of more interest, than the individual particle behavior. According to the forces acting on them, particles can move by 3 transport modes: traction, saltation, and suspension. In a slurry flow, these 3 transport modes, plus the static state, are combined in various proportions to form different flow pattern typologies. Knowledge of these flow typologies is essential in the study of slurry, to analyze and interpret the head losses or heat transfer coefficient results. In the case of sedimentary flows, many studies have focused on these typologies [11,12]. The diversity of the observed regimes and the associated vocabulary makes it difficult to compare these various studies, and thus complicates the overall understanding of the phenomena taking place during the transport of solid particles.

A rather complete work on the subject was recently carried out by Ramsdell \& Miedema [13]. They present the phenomenon of sedimentary transport as a scenario taking place according to the flowrate. They describes 9 various flow pattern typologies, which when the flow rate increased and according to the operating conditions (duct diameter, particles characteristics, fluid characteristics), will follow one another to form a scenario. Depending on the experimental conditions, various scenarios can be obtained, and each one consists of a succession of typologies. First, they
$P \quad$ power dissipated by the flow $[\mathrm{W} / \mathrm{m}]$

SEC specific energy consumption $[\mathrm{J} / \mathrm{g} / \mathrm{m}]$

$\Phi \quad$ volumetric concentration [\%vol]

$\Phi_{\max } \quad$ particle packing factor [\%vol]

$\varphi \quad$ delivered concentration [\%vol]

$\theta \quad$ Shields number [-]

Subscript and superscript

liq liquid phase (water)

sol solid phase (particles)

m mixture distinguish two theoretical types of scenario. The first, which they name laboratory conditions corresponds to a closed circuit without storage volume. In this case, the quantity of solid present in the loop is contained in pipes and distributed uniformly. The working condition is thus a constant spatial concentration. The delivery concentration is thus related to the fixed spatial concentration and the flowrate. The second type, which they name real conditions, corresponds to an open or closed circuit with a very large storage volume. In this case, the spatial concentration in the tank dominates the transport phenomenon in the ducts. In fact, the spatial concentration in the tank (which must be homogeneous) will be transformed into the delivery concentration as the entrance boundary condition of the circuit. The spatial concentration in the circuit is thus related to the delivery concentration and the flowrate. As is very often found, the reality does not correspond to the theoretical perfect cases. The practical cases are thus intermediate between the two. The evolution of the head losses versus flowrate is related to the scenario obtained. In their publication, Ramsdell and Miedema [13] identify 6 different scenarios and present the associated head losses.

To predict the behavior of flowing slurry, models have been proposed to determine the evolution of the flow patterns and the associated head losses. There are numerous models because very often, their domain of validity is very restricted. The different approaches are:

- By empirical correlations: These are based on dimensional analysis, the coefficients of dimensionless parameters $(k, \alpha, \beta, \gamma$ and $\delta$ in Eq. (1)) are identified by experimental data fitting. One of the most useful correlation [11] is based on the following empirical form:

$$
f-f_{w}=k \cdot C^{\alpha} \cdot f_{w}^{\beta} \cdot C_{D}^{\gamma} \cdot\left(\frac{v^{2}}{D \cdot g \cdot\left(\frac{\rho_{s}}{\rho_{l}}-1\right)}\right)^{\delta}
$$

where:

$$
f_{w}=\frac{1}{2} \frac{D}{\rho v^{2}} \frac{-\Delta P}{L}
$$

In this case, the extra work required to impose a given mixture flow rate (friction factor $f$ in Eq. (1)) is compared with the work required for a pure water flow rate at the same mean velocity $\left(f_{w}\right.$ and $v$ in Eq. (2) respectively) and express classically as a function of power law of dimensionless parameters. It should be noted that although this kind of method is very useful and common in engineering, the coefficients have to be tuned for the different flow patterns in order to fit reality. Thus, in the case of Turian's correlation [11], four transition laws were given to suit the 
following flow regimes: stationary bed, saltation, heterogenous and homogenous. These correlation were built using settling slurries and could not be applied to floating slurries even if they had been built using a wide range of dimensional parameters (pipe diameter, solid density, etc): identification procedures would have to be performed, especially for the modified Froude coefficient $\delta$ in order to take into account the change in sign of the term $\left.\frac{\rho_{s}}{\rho_{l}}-1\right)$. To the best of the authors, no equivalent correlation has been published concerning or including floating slurries.

- By models of the effective liquid [14] coupledwith a multi-layer model [5]: In this kind of model, it is supposed that the liquidsolid mixture can be modeled by a stack of layers in which the fluid has different effective properties.

Concerning the calculation of the effective viscosity of the mixture, many alternatives have been proposed, more or less adapted to take into account particles shape, size heterogeneity, the fluid, or the particles arrangement in the flow. Reviews of these different models can be found in $[15,16]$.

This state of the art quotes almost only those works on sedimentary slurry, which are the most. Nether less, there are many industrial flow applications of light particles, for example the ice slurry flow.

Ice slurry [17], which is a flowable phase change material, seems promising for use as a secondary fluid in refrigeration systems [18]. The technology involved, studied for over 20 years, is now mature for industrial use $[19,20]$. Many studies have been conducted on ice slurry flow in tubes [21,22] and in sudden constrictions $[23,24]$. For high flow rates, the assumption of an equivalent homogeneous medium seems sufficient to model the system. Some authors therefore work on the rheology of ice slurries $[25,26]$. However, this homogeneous regime, obtained at high flow rates, is not necessarily needed to transport ice slurries efficiently and to optimize energy efficiency. In fact, ice slurries have great apparent heat capacities and therefore can transport a great deal of energy even at low flow rates. For example, in ice slurry flows, a variety of flow patterns are observed and they depend on the piping system, the operation parameters (different production methods, pipe diameter, ice velocity and concentration). The number of applications is increasing. Ice slurry cooling systems have been installed in buildings for air conditioning purposes, food processing and preservation, and possible future applications (ice pigging, medical applications, artificial snow, fire fighting) [27]. The advantages of this mode of transportation, such as its environment friendliness, the relatively little infrastructure work needed and possibly low operation and maintenance costs, may make it an attractive alternative to other methods. Furthermore, the use of ice slurry fits into a plan of reducing energy consumption, which has to be optimized in all parts of the process, including transportation. Therefore, it is important, not only to optimize heat transfer from ice slurries, but also to find the best operating conditions that will lead to the good transport of ice without energy waste within pumps. The complexity of coolant refrigeration systems (variation in cross-section, flow divider, flow rate variation) creates difficulties in driving these systems [28]. This, combined with a risk of blockage, explains why ice slurry technology is currently used mostly for energy storage and not as a secondary coolant. To encourage this development of ice slurry, it is important to complete former studies and to improve the analysis of the different flow regimes that may occur in these systems. However, prior to thermal analysis, the hydraulic phenomena that would occur in the installation must be understood. It is generally agreed that heating efficiency is a combination of a good heat transfer capacity and an optimized hydrodynamic pattern, to improve forced convection. Moreover, as already mentioned, high flow rates generate high energy costs, which also have to be taken into account in energy balances.

This paper is part of a larger work that aims to optimize the overall operating conditions for the use of ice slurry, in order to enable its industrial use. As in many studies [29]we chose to uncouple the hydrodynamics and heat transfer by working in isothermal conditions, in order to analyze the hydrodynamics of ice slurry in the range of laminar to low turbulent flows. Further studies will deal with the coupled phenomena. Moreover, with ice slurry, the hydrodynamics are difficult to study because of ice melting, which leads to a modification of the concentration and shape of the solid phase [30]; water condensation on pipes also interferes with observations. In order to overcome these difficulties, we propose, like previous authors [31], to study granular model fluids, chosen especially to have the same characteristics as those of ice slurries. The results obtained will be used in isothermal applications too.

The objective of this study is to understand the behavior of a slurry, in an isothermal regime, made up of floating particles and identical to an ice slurry. These characteristics of particles have been little studied. Nevertheless, it is clear that the behavior of this kind of slurry will differ from that of sedimentary slurry. For example, the transport of saltation will not be present because the particle density is lower than that of the fluid.

A specific device has been designed and used to observe the flow typologies, measure the head losses and determine the flow concentration. Section 1 is devoted to the presentation of the experimental setup, the methods used, the associated instrumentation and the choice of particles. The experimental results obtained are presented in Sections 2.1 and 2.2. The main objective of this study is to determine the conditions that minimize the energy consumed by installations using such fluids. These measurements are made with the final goal of determining the transport energy efficiency. The best combinations that provide the energetic optimum are described in Section 2.3. The results obtained are valid for other slurries (light particle applications).

\section{Materials and method}

\subsection{Presentation of the experimental loop}

Fig. 1 is a schematic diagram of the experimental apparatus. It consists of a closed loop in which a mixture of water and solid particles circulates thanks to a centrifugal pump (Grundfos CHV2). The section of passage in the enclosed impeller is $3 \mathrm{~mm}$, which is sufficient for the circulation of the particles used. The pump rotation speed, controlled by a frequency converter, enables the mixture flow rate to be varied without using a valve. This avoids abrupt section reduction and consequently particle accumulation and plug generation. The measuring section is composed of a 4-m long

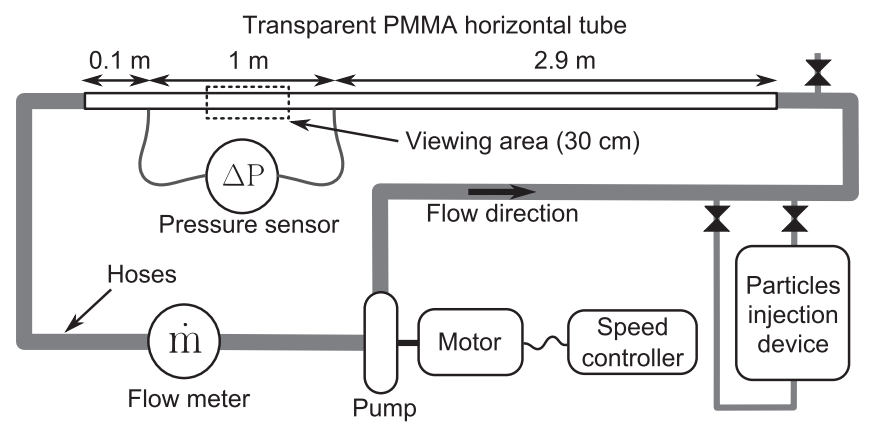

Fig. 1. Schematic of experimental setup. 
transparent PMMA pipe, with a $30 \mathrm{~mm}$ diameter. The first $2.9 \mathrm{~m}$ is required to ensure the hydrodynamic establishment of a monophasic flow according to the classic correlation in laminar and turbulent regimes [32] in Newtonian fluids. (In the case of liquid-solid fluids, to our knowledge, there is no study on the flowestablishment length). The last $0.1 \mathrm{~m}$ is present to limit upstream effects.

Hoses of the same diameter connect the pipe to the different elements of the apparatus (pump, flow meter and particle injection device). In such systems, the flow in a local section is clearly influenced by the behavior of the mixture in the rest of the loop. In order to avoid geometrical influences, the setup is designed without a storage tank, to ensure perfect continuity in the cross-section throughout the circuit (except inside the pump and the flow meter). The flow conditions are thus the same everywhere in the loop. The volumetric concentration $(\Phi)$, regulated at the beginning of the experiment, is thus constant in all sections of the loop. This volumetric concentration should not be confused with the delivered concentration $(\varphi)$, as discussed below.

\subsection{Characteristics of the mixture}

There are many studies on the transport of granular media, but the vast majority concern sediment particles: the solid phase is heavier than the liquid phase and thus stratifies at the bottom of the pipes. In the case of ice slurry the buoyancy acting on solid inclusions leads to the stratification of particles at the top of pipes. These mixtures are much rarer than sediment slurries and have been less studied. However, this inversion in the density ratio also affects particle transport by changing the influence of inertial forces. For example, with a density ratio of 0.9 , saltation transport is not observed, while it is dominant in flows with a high density ratio like Aeolian sand transport $\left(\rho_{\text {sand }} / \rho_{\text {air }}>2000\right)$ [33].

The first objective of this study was to obtain a model fluid as close to ice slurry as possible. For this reason we worked with water at room temperature as the liquid carrier $\left(\mu_{\text {liq }}=0.91 \pm 0.04 \mathrm{mPa} \mathrm{s}, \quad \rho_{\text {liq }}=997 \pm 1 \mathrm{~kg} \mathrm{~m}^{-3}\right)$. The choice of floating solids in water available in granular state is poor. To approximate the density of ice $\left(\rho_{\text {ice }}=917 \mathrm{~kg} \mathrm{~m}^{-3}\right)$, polypropylene seemed to be a good choice. This material had already been selected by Stutz et al. [34] for the same purpose. We thus used a polypropylene powder (ICORENE4014R+) produced by Icopolymer and made from crushed pellets at very low temperature. The density was measured using a helium pycnometer(Micrometrics AccuPyc 1330) and was found to be $\rho_{\text {sol }}=907 \pm 1 \mathrm{~kg} \mathrm{~m}^{-3} .95 \% \mathrm{vol}$ of the particles were black, in order to obtain a good contrast and to observe the overall distribution of the particles inside the flow in the best conditions possible; $5 \% \mathrm{vol}$ of the particles were white in order to track a single particle and observe its individual path.

To represent the variety of crystal sizes $[19,35]$ the powder was sieved. Sieves of $310 / 560 / 760 / 910 \mu \mathrm{m}$ were used to select three size ranges. After sieving, the size dispersion was measured by a laser particle sizer (Malvern Mastersizer 2000). The results are shown in Fig. 2.

Diameters were measured before and after many hours of circulation in the experimental loop. No size variation was observed, confirming that the centrifugal pump did not damage the particles. The particles used were not completely spherical and their surfaces were rough as illustrated in Fig. 3. The ratio of length to width was close to 1.5 .

The packing factor, i.e. the maximum volumetric concentration, is an important parameter in particle stratification. For a random packing of monodisperse spherical particles, the packing factor fluctuates in the range of 0.64 (close packing factor [36]) to 0.55

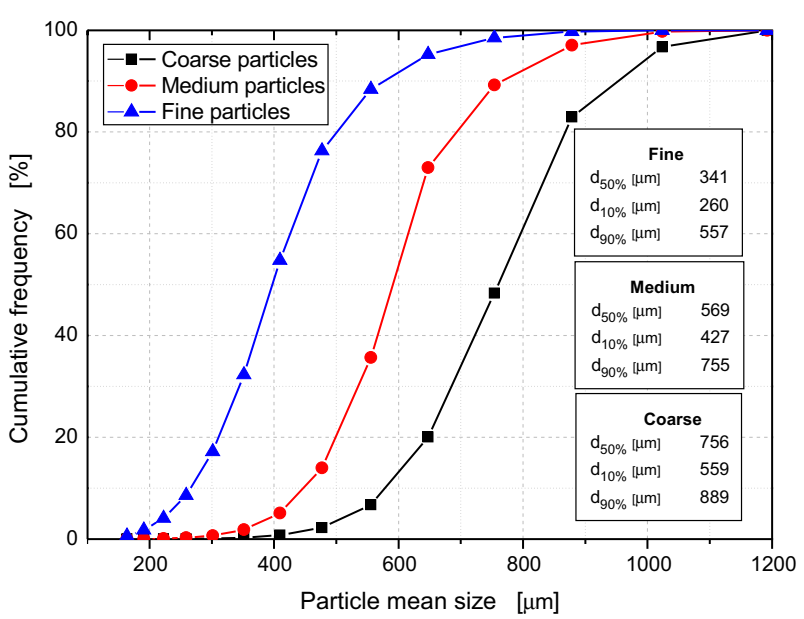

Fig. 2. Size distribution of particle diameters.

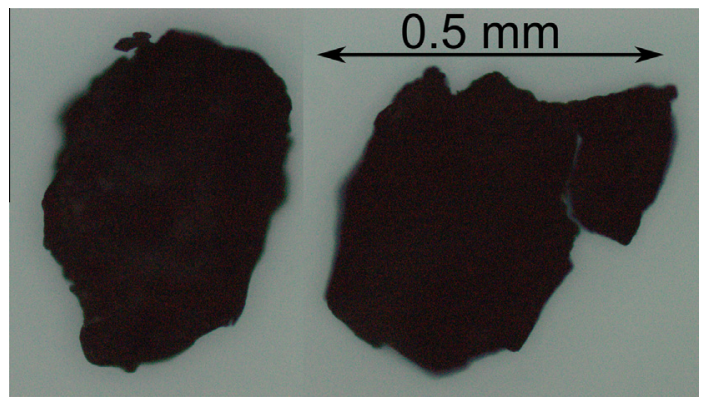

Fig. 3. Photographs of medium particles.

(loose packing factor [37]). The packing factor for the polypropylene particles used was determined by measuring the solid mass needed to fill a specific volume. The apparent weight acting on the particles (compression forces) affects the volume filled. Under our experimental conditions, the packing factor was $\Phi_{\max }=0.5 \pm 0.03$. The non-sphericity, roughness and light polydispersion of the particles led to a lighter compacity than might be expected.

\subsection{Particle injection methodology}

The insertion of particles in the experimental loop is not easy, particularly in the case of floating particles. For sedimentary particles, it is possible to use a storage tank. At rest, particles sediment at the bottom of the tank and a classic stirrer is sufficient to put in them into suspension. A fraction of the fluid can be extracted and put into circulation in the loop [38].

When floating particles, are at rest, they rise and accumulate at the interface between the liquid and air. If a stirrer is used to put them into suspension, it has to create eddies on the surface to catch the particles near the surface. These eddies inevitably lead to air bubbles with particles.

The mixture obtained can not thus be sent directly into the loop. Especially as the particles used tend to gather together around air bubbles, which become the site of formation of particle flocs. We devised an assembly that enabled the loop to be loaded with solid first, before working in a closed loop (laboratory conditions according to [39]).

The particle injection device was used to control the amount of solid particles added inside the loop: part of the water circulating was withdrawn using a filter; then a quantity of solids was mixed 
with it and re-injected into the loop in order to obtain the chosen static volumetric concentration $(\Phi)$. During the injection, care was taken to monitor the amount of injected particles and to avoid introducing air bubbles. The few remaining bubbles were removed by adjusting the solubility (with temperature and pressure) of air in water [40].

\subsection{Metrology and protocol}

A Coriolis mass flow meter (E+H Promass 83I) with a 6-mm-diameter flow section was used to measure the mixture mass flow rate $\left(\dot{m}_{m}\right)$ (full range: $0-0.5 \mathrm{~kg} \mathrm{~s}^{1}$, accuracy: $0.1 \%$ ). The flowmeter was also used to evaluate the mixture density $\left(\rho_{m}\right)$ (accuracy: $0.5 \mathrm{~kg} / \mathrm{m}^{3}$ ). This was necessary to calculate the delivered concentration $(\varphi)$, as explained below.

The pressure drop $(\Delta P)$ was measured with a differential pressure sensor ( $E+H$ Deltabar PMD75) (full range $0-1 \mathrm{kPa}$, accuracy $1.5 \mathrm{~Pa}$ ). The distance between the pressure gauges was equal to $1.00 \mathrm{~m}$.

All sensors were connected to a personal computer via a DAQ (Agilent 34970A) allowing data acquisition with a frequency of $2 \mathrm{~Hz}$. The results shown are the average of $10 \mathrm{~min}$ of measurement.

The test section was built with a transparent pipe (PMMA) to allow the flow to be visualized. A camera (Point Grey Chameleon) was used to record the side view of flows obtained at $3.2 \mathrm{~m}$ from the entrance. The camera was positioned approximately $50 \mathrm{~cm}$ in front of the tube so as to observe a tube length of $10 \mathrm{~cm}$. The obtained depth of field was then of the order of the tube diameter. The videos thus recorded enable the flow patterns to be determined according to different parameters.

Before each acquisition, the pump was switched on and operated at its maximum speed for 5 min to ensure a homogeneous concentration throughout the loop. Then the flow was abruptly stopped in order to generate a flat particle bed at the top of the tube. Starting from zero, the flow rate was progressively increased to observe its influence. Prior to each measurement, a stabilization/establishment time of $30 \mathrm{~min}$ was observed. Using a valve to control the flow rate would cause a localized accumulation of particles in front of it, increasing the risk of blockages and invalidating the assumption of a constant concentration throughout the loop. The flow rate was thus controlled by increasing the pump rotational speed.

The maximum volume fraction of solid studied was $25 \% \mathrm{vol}$ with a resolution of $5 \% \mathrm{vol}$. At high concentrations (15-25\%vol), blockages appeared at low flow rates at the top of the vertical sections. At this point, particles were crowded and could form clusters several $\mathrm{cm}$ thick. Until the rotational speed of the pump was sufficient to exert a pressure able to expel the plug, no flow circulated.

\section{Experimental results}

\subsection{Presentation of results}

When a fluid is monophasic, hydraulic flows are described using dimensionless numbers, such as the Reynolds number and the Darcy friction number. In the case of slurry there are various dimensionless numbers to characterize the different phenomena (Pipe Reynolds number, Particle Reynolds number, Generalized Reynolds number, Froude number, Rouse number, etc.). In view of the literature, it is difficult to favor one over anothers. Moreover the many constructions of these dimensionless numbers in articles make it difficult to compare the results obtained. For this reason, we chose to present our results in a dimensional way, mainly using the mixture mass flow rate measured. However, the flow regime (laminar or turbulent) must be identified. In the case of pure water this transition appears for a critical Reynolds number of around 2300. In our experimental conditions, it corresponds to a mass flow rate of $55 \mathrm{~g} / \mathrm{s}$ for pure water. The maximum mass flow rate studied ( $500 \mathrm{~g} / \mathrm{s}$ ) corresponds to a Reynolds number of around 20,000 for pure water.

\subsection{Flow patterns}

The competition between buoyancy, weight and the hydrodynamic forces acting on the particles leads to different flow patterns. The determination of the flow pattern is very important because the behavior of the slurry greatly depends on it. Referring to the analysis proposed by Ramsdell \& Miedema [13], our observations were made in laboratory conditions, i.e. at constant static concentration. Using the recorded videos, the flow pattern for each experiment was determined. In our flow conditions, only four flow patterns were distinguished, illustrated in Fig. 4, separated by two remarkable transitions. These patterns and transitions are discussed below. The maps of the flow patterns obtained depending on the mass flow rate and the volumetric concentration are presented in Figs. 5-7, for the different particle diameters studied.

\section{(Pattern I) No solid motion (Fig. 4A)}

The flow rate is too low to enable the motion of particles, which are deposited by stratification in the upper part of the pipe. In this study, stratification generates a flat bed due the protocol used and already described. The shear stress caused by the fluid flow on the bed interface is not high enough to extract particles. This stress is communicated to the wall by the different layers of particles. In our case the friction between the wall and the bed was high enough to ensure a no slip condition. The concentration in the bed formed corresponds to the maximum volumetric concentration. The bed can be compared to a microporous medium. In our range of particle diameter, the drag in the bed [41] is high enough, compared to the drag in the free cross-section, to make the hypothesis of an impermeable bed. Water then flows in the truncated section, with a higher velocity than in the case of no particles.

\section{(Transition I $\rightarrow$ II) Motion threshold}

When the flow rate reaches a critical value, the shear stress on the first layer of particles is high enough to extract and set in motion a few particles. They then roll or slide on the surface of the bed which stays fixed.
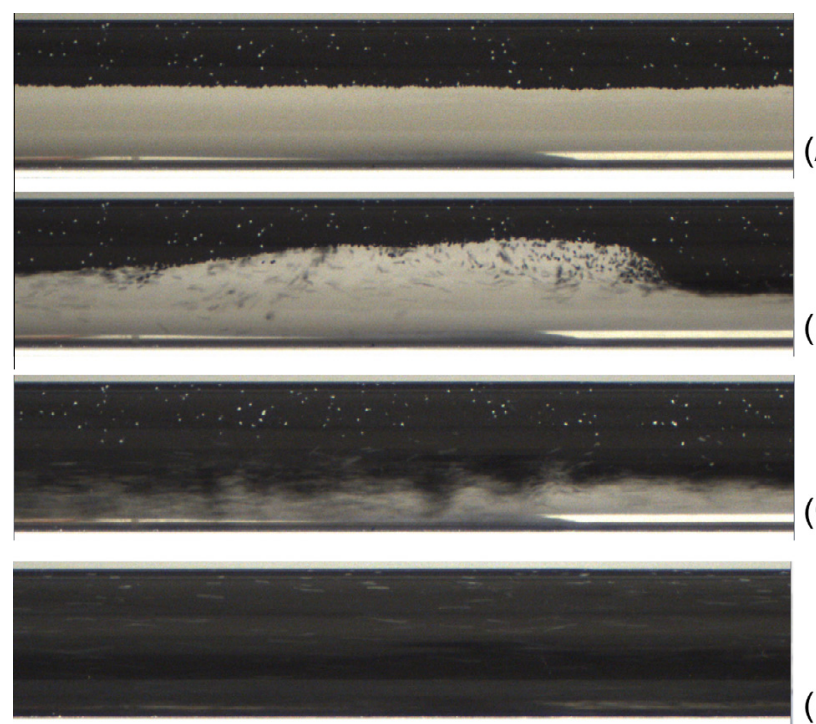

Fig. 4. Photographs of flow patterns at $\Phi=15 \%$ vol for medium particles. (A) No solid motion. (B) Wavy bed load. (C) Flat bed load. (D) Fully suspended. 


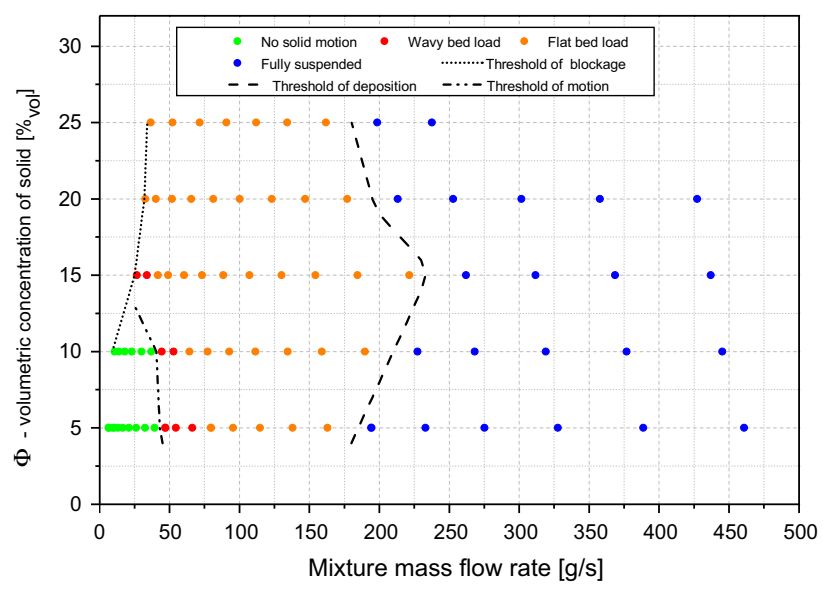

Fig. 5. Flow pattern maps in term of the volumetric concentration versus mass flow rate for fine particles.

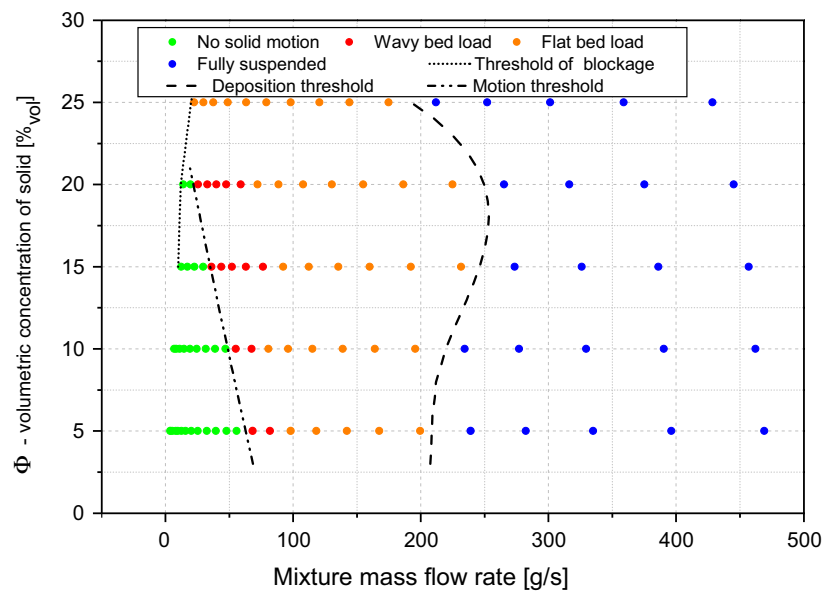

Fig. 6. Flow pattern maps in term of the volumetric concentration versus mass flow rate for medium particles.

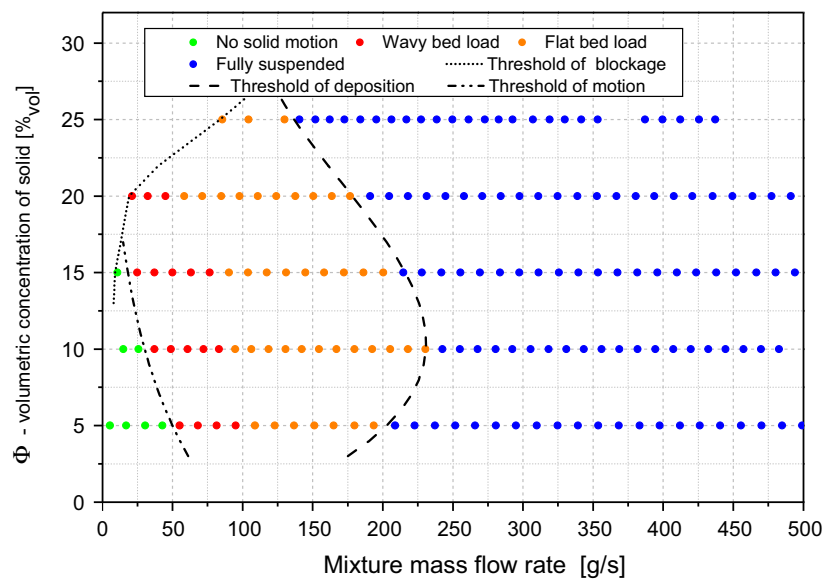

Fig. 7. Flow pattern maps in term of the volumetric concentration versus mass flow rate for coarse particles.

This threshold in circular pipes has been studied for laminar flow with particles heavier than the liquid phase, by Ouriemi et al. [42]. The Shields number (Eq. (3)), a dimensionless wall shear stress, is the key number to determine it. For laminar flow, they found a critical value, corresponding to the onset of motion, of $\theta_{c}=0.12$.

$$
\theta=\frac{\tau_{w}}{\left(\rho_{\text {liq }}-\rho_{\text {sol }}\right) g d}
$$

In turbulent flow, the determination of the wall shear stress is not simple. Peysson et al. [43] proposed a model, using geometrical calculation and classical correlation, to calculate the Shields number in pipe turbulent flow. Using this method, we determined the critical Shields number associated with the threshold of motion. The calculated values are shown in Table 1.

The critical Shields number in our condition was found to be lower than the value determined in laminar flow [42]. This is partially due to the fluctuation of the wall shear stress over time, generated by the turbulence, which causes very high shear stress temporarily. In our range of study, the threshold value decreases with the increase in particle diameter. This leads to the counter intuitive statement that, in our conditions, it is not easier to set smaller particles in motion. This behavior is due to the fact that coarse particles exceed the thickness of the viscous sub-layer, increasing the constraints they experience. Soulsby \& Whitehouse [39] have proposed a correlation to estimate the critical value of the Shields number (Eq. (4)) for sand grains. The value obtained with this correlation is reported in Table 1 and fits well with our results.

$$
\begin{aligned}
& \theta_{\mathrm{c}}=\frac{0.30}{1+1.2 \mathrm{D}^{*}}+0.055\left[1-\mathrm{e}^{-0.02 \mathrm{D}^{*}}\right] \\
& \mathrm{D}^{*}=d\left(\frac{\rho_{\mathrm{liq}}\left|\rho_{\mathrm{liq}}-\rho_{\mathrm{sol}}\right| \mathrm{g}}{\mu_{\mathrm{liq}}^{2}}\right)^{1 / 3}
\end{aligned}
$$

(Pattern II) Wavy bed load (Fig. 4B)

The more the flow deviates from the motion threshold, the more particles are set in motion. A sliding layer of particles emerges at the surface of the fixed bed. Under this layer, a few particles are found traveling by suspension. The flow is then able to shape the fixed bed by erosion. Instability of the flow is amplified and deforms the original flat bed creating bed forms $[44,45,40]$. With alternating saltation and stratification, particles are alternately in the fixed bed and in the moving layer, allowing the dunes to shift forward.

(Pattern III) Flat bed load (Fig. 4C)

When the flow rate increases, bed-forms are eroded by the flow, becoming smaller and smaller until they disappear completely. During this phase, more and more particles travel by suspension. This suspension layer represents the lower layer of particles in the tube and tends to grow toward the top of the tube. The intermediate layer (where transport is mainly due to rolling and saltation) also tend to shift to the upper part of the tube until the upper layer (the fixed bed) becomes thinner and thinner, until only the finest moving layer (of the height of the particle diameter) remains. It should be noted that this is one possible scenario, but the transition from pattern 1 to pattern 3 can differ in terms of particles layers (suspending, rolling-salting, and no-moving) behavior.

(Transition III $\rightarrow$ IV) Deposition threshold

When the flow rate reaches a second critical value, the hydrodynamic forces are high enough to suspend all the particles.

Table 1

Critical Shields numbers

\begin{tabular}{llll}
\hline$d[\mu \mathrm{m}]$ & 341 & 569 & 756 \\
\hline$\theta_{c}^{\text {exp }}$ & $0.08 \pm 0.01$ & $0.06 \pm 0.01$ & $0.04 \pm 0.01$ \\
$\theta_{c}^{\text {th }}($ Eq. (4)) & 0.063 & 0.045 & 0.038 \\
\hline
\end{tabular}


The moving layer composed of rolling particles disappears. Particles close to the wall, found at high concentration, are now moving by suspension and are not in constant contact with the wall of the pipe. Our results indicate a non-linear evolution of this threshold as a function of the concentration. Low and high concentration slurries appear to be more easily placed in suspension. This behavior has already been observed for sand slurries [46]. The non-monotonic evolution, with particle size, of this threshold makes it difficult to study it from our results. (Pattern IV) Fully suspended flow (Fig. 4D)

All particles are transported by suspension, although a clear heterogeneity in the concentration profile can be observed. When the flowrate increases, the mixture tends to be homogeneous.

\subsection{Pressure drop}

The pressure drop measured, within the range of the flowmeter, is presented in Figs. 8-10 for the three size ranges of particles. The volumetric concentration is differentiated by color, while the flow pattern is differentiated by the shape of the marker. Thereafter, the same notation system is used.

The experimental setup and the pressure sensor were qualified with pure water, by controlling the reproducibility and comparing the results with reference values calculated with a classic

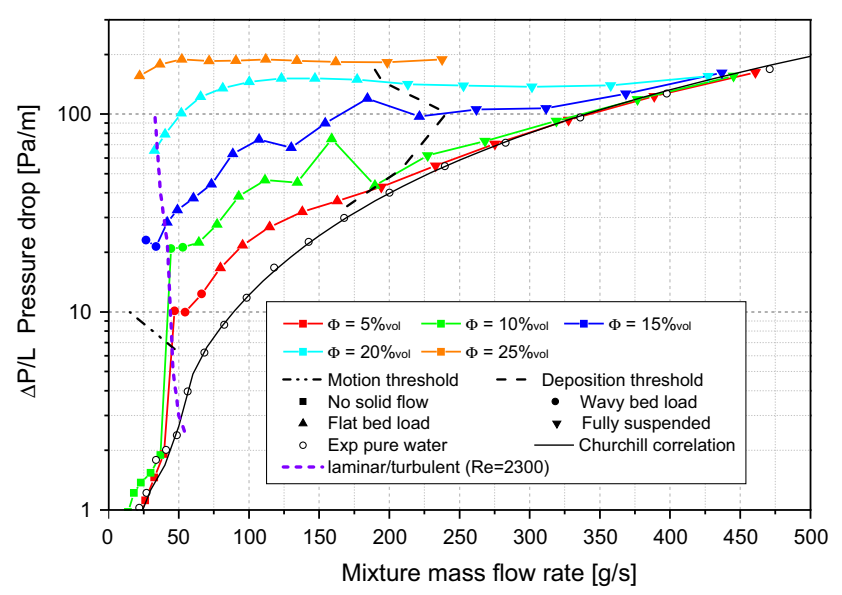

Fig. 8. Flow pattern maps in term of the volumetric concentration versus mass flow rate for fine particles.

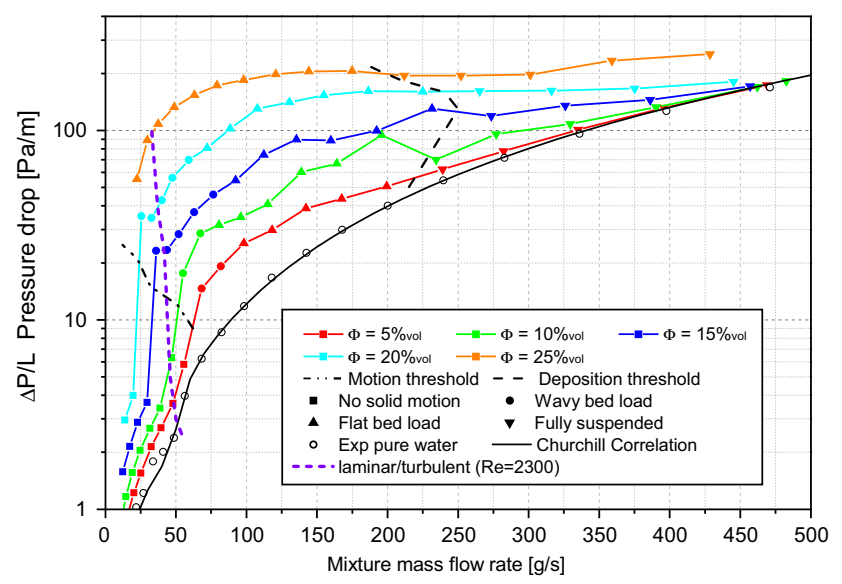

Fig. 9. Flow pattern maps in term of the volumetric concentration versus mass flow rate for medium particles.

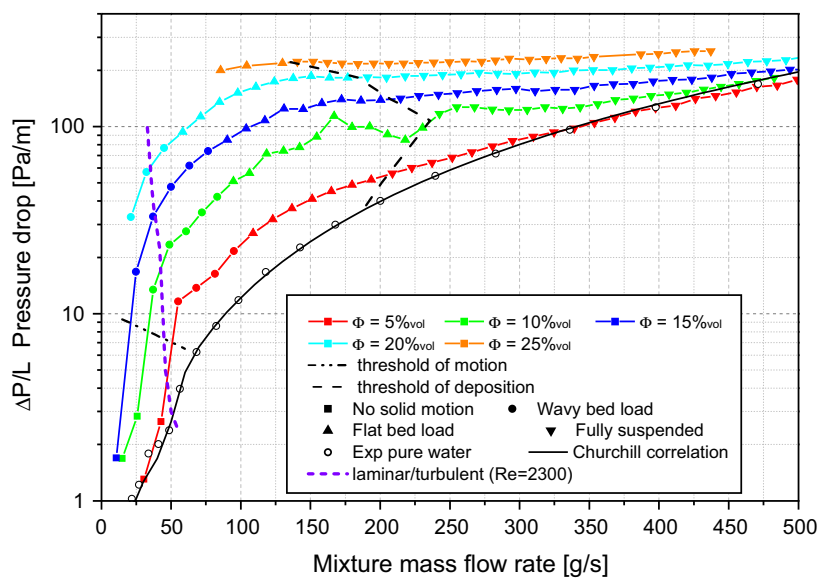

Fig. 10. Flow pattern maps in term of the volumetric concentration versus mass flow rate for coarse particles.

correlation. The results presented in Figs. 8-10 agree with the correlation proposed by Churchill [47] for smooth pipes in both laminar $\left(\dot{m}_{m}<55 \mathrm{~g} / \mathrm{s}\right)$ and turbulent $\left(\dot{m}_{m}>55 \mathrm{~g} / \mathrm{s}\right)$ regimes.

For very low flow rates, when the flow is laminar, the solid phase is not set in motion. The stratification of particles reduces the cross section and leads to a higher mean velocity of the liquid, thus increasing the pressure drop compared to pure water. This phenomenon is clearly visible for the medium particles in Fig. 9, from 0 to $50 \mathrm{~g} / \mathrm{s}$.

When the threshold of motion is reached, a large increase in the pressure drop can be observed. This phenomenon appears almost simultaneously with the transition from laminar to turbulent flow. This is well illustrated by the iso-Reynolds curve plotted in Figs. 810. A constant Reynolds number value of 2300 was chosen for the transition from laminar to turbulent flow, and thanks to the hydraulic diameter definition and a constant packing factor of 0.5 , the corresponding transitional mass flow mixture was estimated.

It is therefore difficult to know the exact impact that each phenomenon has on the pressure drop. During the wavy flow pattern, the differential pressure measured is not constant in time. This is due to the presence of bedforms, modifying the local average velocity in space and time and leading to a transfer of energy between kinetic and static pressure energies. To determine the head losses we had to average our measurements over a long time period (more than 10 , depending on the presence of dunes). This fluctuation in static pressure was successfully use to determine the frequency of the dune transport [40]. Bedforms act as a series of singular head losses, braking the spatial establishment of the flow and generating more pressure drop which is added to the regular pressure drop.

Once bedforms are smoothed by erosion, the evolution of the pressure drop does not monotonically increase as one might expect. In fact, sometimes, several local minimums can be perceived. This is especially true for a concentration of $10 \%$, whatever the size of the particles. It can be explained by the appearance of instabilities still poorly understood and difficult to observe [48].

When the fully suspended flow pattern is obtained, the difference in the head losses between pure water and slurry is reduced when flow rate is increases. For the highest flow rates, the difference is minimal or even negative. Therefore, particles do not necessarily act as a viscosity increaser, although this is commonly accepted for slurries. In turbulent flow, particles could act as a drag reducer [49], as was observed by Knodel et al. [35] for high flow rates with coarse ice slurry. This can be compared to the re-laminarization described by Niezgoda-Zelasko \& Zelasko [2] for ice slurry flow. 


\subsection{Delivered concentration}

As mentioned previously, the volumetric concentration $(\Phi)$ in the setup is controlled by the amount of particles added during the injection protocol and represents the amount of particles in a specific volume fraction.

However, depending on the flow rate (and thus on the flow pattern), only a fraction of these particles are transported. To quantify the fraction of solid carried by the fluid, we use the delivered concentration $(\varphi)$, defined as the volumetric flow rate of solids compared to the total volumetric flow rate (Eq. (5)). In our case, since we are working in a closed loop, the delivered concentration is spatially constant in the setup when the steady state is reached.

$\varphi=\frac{\dot{V}_{s o l}}{\dot{V}_{m}}$

The cross section diameter in the Coriolis mass flowmeter is $6 \mathrm{~mm}$, which is very small compared to the $30 \mathrm{~mm}$ of the rest of the setup. The mixture velocity is thus very high in the measurement section of the flowmeter ensuring the complete transport of the solid phase with a minimal slip velocity (difference in velocity between solid and liquid phases). Assuming a zero slip velocity, this generates an equivalence between the volumetric concentration and the delivered concentration inside the flowmeter. The volumetric concentration, calculated using the measurement of the density inside the flowmeter (Eq. (6)), corresponds therefore to the delivered concentration inside the setup.

Special attention was paid to the air entrapped between or at the surface of the particles. The mixture was introduced with care in order to limit the amount of air injected into the setup. The device was then heated to $45^{\circ} \mathrm{C}$ temporarily and its pressure lowered to 1 bar (whereas tests were carried out at 2 bars) to ensure a very low solubility of air in water. Most air bubbles were then discharged through a vent. The small amount of air not removed by this procedure dissolved completely when the test conditions were established, and it was verified visually that even very small air bubbles attached to particles disappeared.

$\varphi=\Phi_{\text {flowmeter }}=\frac{\rho_{\text {liq }}-\rho_{m}}{\rho_{\text {liq }}-\rho_{\text {sol }}}$

Our results are presented in Figs. 11-13. As expected, until the motion threshold is reached, the delivered concentration is zero. It then it increases with the mixture flow rate. The maximum value corresponds to the volumetric concentration, and is reached asymptotically. One can notice that there are small difference between the

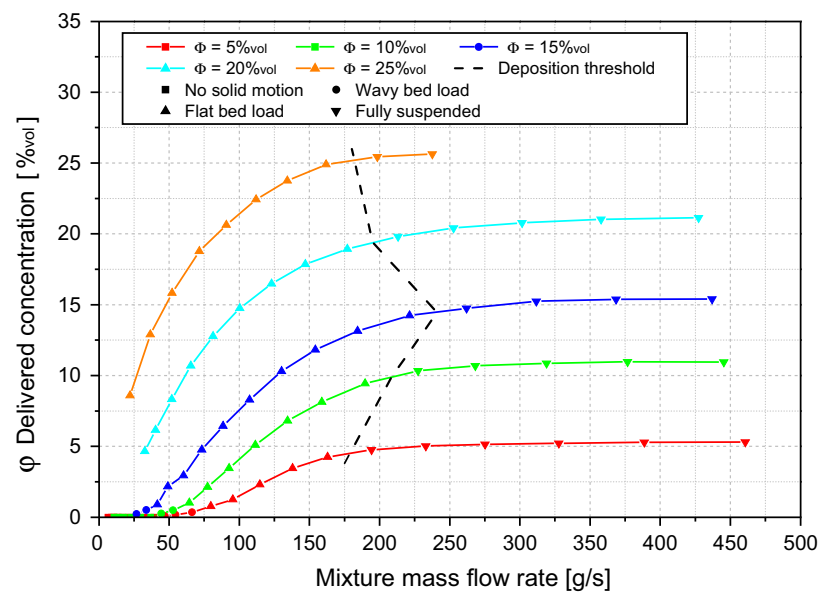

Fig. 11. Flow pattern maps in term of the volumetric concentration versus mass flow rate for fine particles.

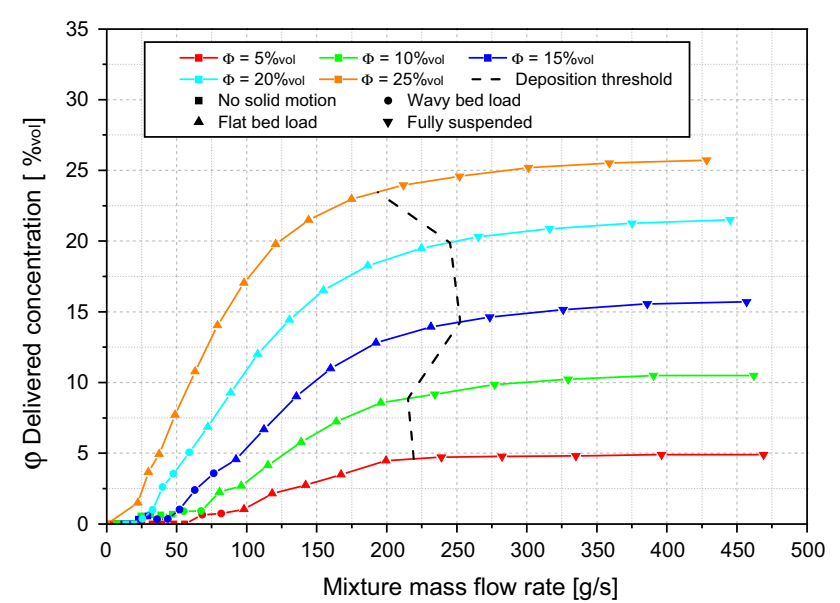

Fig. 12. Flow pattern maps in term of the volumetric concentration versus mass flow rate for medium particles.

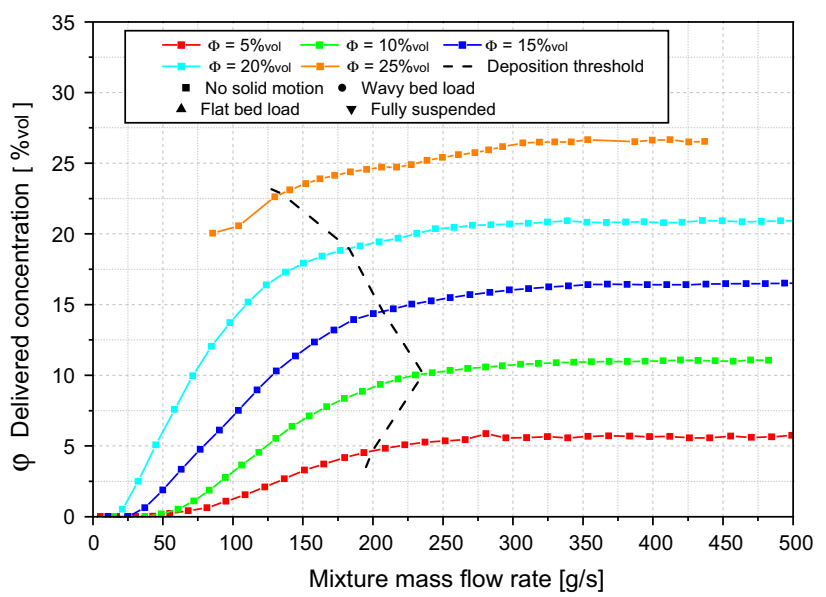

Fig. 13. Flow pattern maps in term of the volumetric concentration versus mass flow rate for coarse particles.

expected volumetric concentration and the asymptotic value of the measured delivered concentration (less than $10 \%$ in the worst case). These can be mainly explained by two reasons: first, the fact that the measurement is always greater than the threshold may be due to an over estimation of the volume of our device. The second reason is directly linked to our injection process, which cannot ensure that all the particles are injected: a small amount may remain stuck to the walls of the injection system. The particle size, in the range studied, does not have a significant impact on the evolution of this parameter.

\subsection{Transport efficiency}

Based on the results presented above, it is possible to calculate the specific energy consumption (SEC) to transport solid particles. The SEC is linked to the pressure drop and the delivered concentration as expressed in Eq. (7). In a complete process application, the efficiency of the pumping system, which changes with the concentration [26], should be taken into account.

$\mathrm{SEC}=\frac{P}{\dot{m}_{m}}=\frac{\Delta P}{L \varphi \rho_{\text {sol }}}$

In order to optimize the energy of a system, the SEC must be as low as possible. Figs. 14-16, where the SEC is plotted versus the mass flow rate, enable the optimum conditions for the transport 


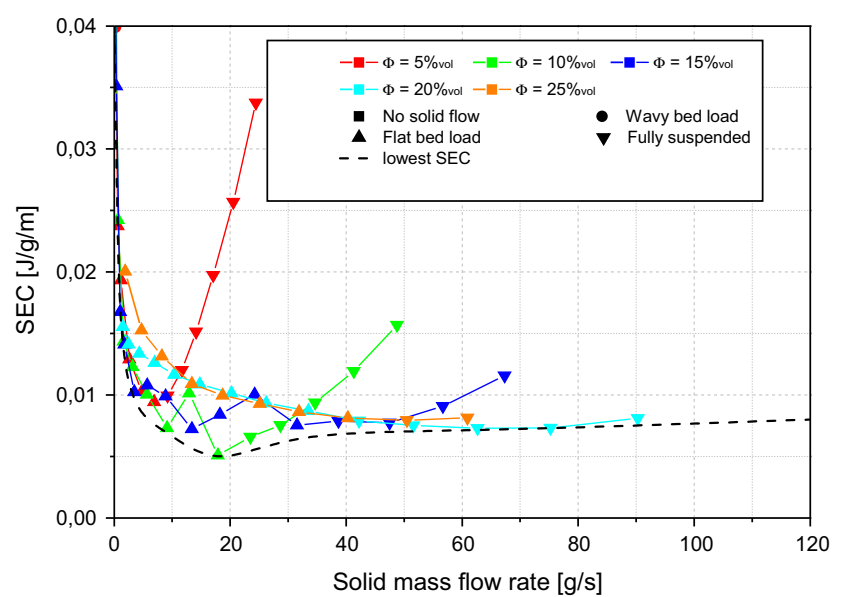

Fig. 14. Specific energy consumption versus solid mass flow rate for fine particles.

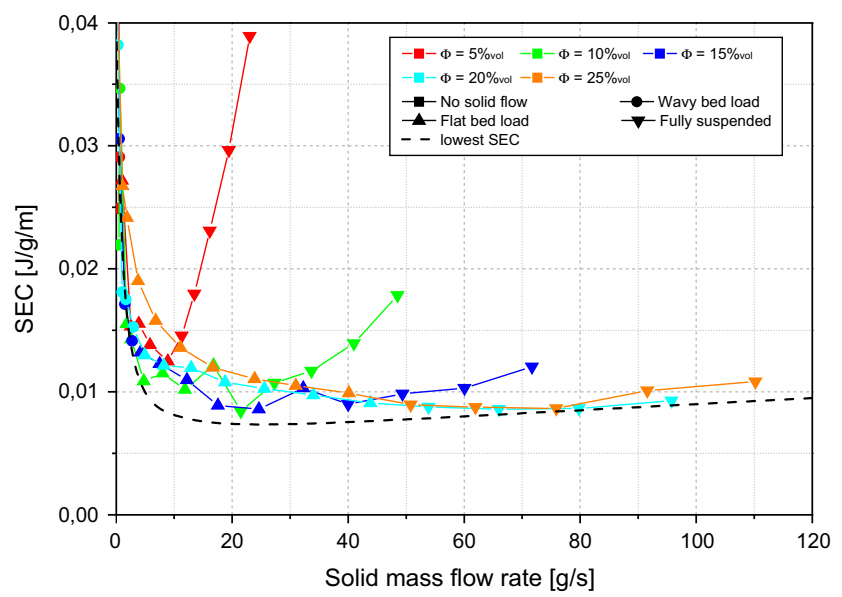

Fig. 15. Specific energy consumption versus solid mass flow rate for medium particles.

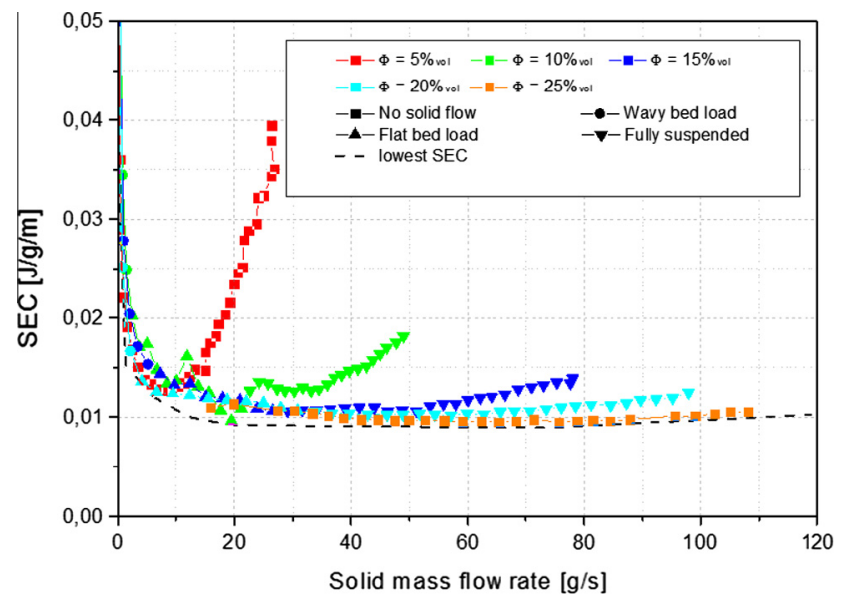

Fig. 16. Specific energy consumption versus solid mass flow rate for coarse particles.

of the solid to be determined. Different series are plotted as a function of the solid concentration. These lines are used to estimate the curve of the best SEC, which would be obtainable if it was possible to vary the concentration of solid.
It can be seen that smaller particles enable better transport efficiency (the SEC is around $20 \%$ for $d=341 \mu \mathrm{m}$ compared to $d=756 \mu \mathrm{m}$ ). The concentration of solid seems to be an interesting parameter to control since, depending on the mass flow rate required, the concentration needed to obtain the best SEC varies. In fact, for a low solid outflow, we should work with a low concentration, and vice versa. In all cases, the best operating point is always not far from the deposition threshold.

\section{Conclusion}

The aim of the present experimental investigation was to find the best operating conditions for transport slurries made of buoyant particles in water, taking into account the flow patterns. The results obtained with a model fluid composed of a polypropylene suspension in water yielded several clues to reach this objective:

In the range of 0.4 to $0.8 \mathrm{~mm}$, the size of the solid particles has an impact on the transport efficiency. For "manufactured slurries", such as ice slurries, the technology used to generate them must be mastered in order to obtain the smallest particles optimizing the performance of transportation.

Devices that can control the concentration are also required, since they would improve the efficiency of non-constant load systems.

The best transport efficiency is reached at the beginning of the fully suspended flow, around the deposition threshold. There is no need to obtain a homogeneous suspension. Unfortunately, it is often recommended to work at a higher flow rate to ensure no dysfunction. A better understanding of the causes of malfunction, leading to blockages, would certainly help to work closer to this nominal point.

To complete this study, the influence of the pipe diameter, mainly to obtain solid transport in a laminar regime, should be investigated: appropriate modifications of the loop are in progress.

Observations during these experiments highlighted phenomena that could be interesting for further study. We are currently performing more measurements with wavy bed loads to understand and predict the characteristics of the bedforms obtained. The curious evolution of the pressure drop at $\Phi=10 \% \mathrm{vol}$ and around $\dot{m}_{m}=200 \mathrm{~g} / \mathrm{s}$, obtained for all particle diameters, is also a range of experimental conditions that will be studied in more detail. To fully describe the energy efficiency of such a system, the performance of the final exchanger should be taken into account. Numerous studies have been carried out on this subject; in a future publication we will add to the current knowledge by comparing the efficiency of different exchanger geometries for laminar and low turbulent flows.

\section{Acknowledgements}

We would like to thank J. Delmas for technical assistance and $\mathrm{H}$. Joubert for her help in writing of paper. This work was undertaken within the framework of the project PERLE2 funded by the French region of Pays de la Loire and supervised by Dr. C. Castelain and Prof. T. Brousse

\section{References}

[1] J. Wu, L. Graham, S. Wang, R. Parthasarathy, Energy efficient slurry holding and transport, Miner. Eng. 23 (2010) 705-712.

[2] B. Niezgoda-Zelasko, J. Zelasko, Melting of ice slurry under forced convection conditions in tubes, Exp. Therm. Fluid Sci. 32 (2008) 1597-1608, http:// dx.doi.org/10.1016/j.expthermflusci.2008.05.002.

[3] M. Vaezi, A. Katta, A. Kumar, Investigation into the mechanisms of pipeline transport of slurries of wheat straw and corn stover to supply a bio-refinery, Biosyst. Eng. 118 (2014) 52-67. 
[4] M. Ouriemi, P. Aussillous, E. Guazzelli, Sediment dynamics. Part 1. Bed-load transport by laminar shearing flows, J. Fluid Mech. 636 (2009) 295-319, http:// dx.doi.org/10.1017/S0022112009007915.

[5] P. Doron, D. Barnea, Pressure drop and limit deposit velocity for solid-liquid flow in pipes, Chem. Eng. Sci. 50 (10) (1995) 1595-1604.

[6] K. Wilson, G. Addie, A. Sellgren, R. Clift, Slurry Transport Using Centrifugal Pumps, 2006.

[7] J. Schaan, R. Summer, R. Gillies, C. Shook, The effect of particle shape on pipeline friction for newtonian slurries of fine particles, Can. J. Chem. Eng. 78 (2000) 717-725.

[8] L. Pullum, D. McCarthy, Ultra High Concentration and Hybrid Hydraulic Transport Systems, 1993.

[9] T. Asim, R. Mishra, L. Kollar, S. Pradhan, Optimal sizing and life-cycle cost modelling of pipelines transporting multi-sized solid-liquid mixtures, Int. J. Press. Vessels Pip. 113 (2014) 40-48.

[10] P. Lovalenti, J. Brady, The hydrodynamic force on a rigid particle undergoing arbitrary time-dependent motion at small reynolds numbers, J. Fluid Mech. 256 (1993) 561-605.

[11] R. Turian, T. Yuan, Flow of slurries in pipelines, AIChE J. 23 (3) (1977) 232-243, http://dx.doi.org/10.1002/aic.690230305.

[12] P. Doron, D. Barnea, Flow pattern maps for solid-liquid flow in pipes, Int. J Multiph. Flow 22 (2) (1996) 273-283.

[13] R.C. Ramsdell, S.A. Miedema, An overview of flow regimes describing slurry transport, in: WODCON XX: The Art of Dredging, 2013, pp. 1-16.

[14] A. Einstein, Effect of suspended rigid spheres on viscosity, Ann. Phys. 19 (1906) 289-306.

[15] V. Vand, The rheology of suspensions of solid particles, Proc. Roy. Soc. A 466 (2010) 1201-1228, http://dx.doi.org/10.1098/rspa.2009.0445.

[16] D. Jeffrey, A. Acrivos, The rheological properties of suspensions of rigid particles, AIChE J. 22 (1976) 417-432, http://dx.doi.org/10.1002/aic.690220303.

[17] M. Kauffeld, M. Kawaji, P. Egolf, Handbook on Ice Slurries - Fundamentals and Engineering, 2005.

[18] I. Chatti, A. Delahaye, L. Fournaison, J. Petitet, Benefits and drawbacks of clathrate hydrates: a review of their areas of interest, Energy Convers. Manage. 46 (9-10) (2005) 1333-1343, http://dx.doi.org/10.1016/j.enconman.2004.06.032.

[19] A. Delahaye, L. Fournaison, J. Guilpart, Characterisation of ice and THF hydrate slurry crystal size distribution by microscopic observation method, Int. J. Refrig. 33 (8) (2010) 1639-1647, http://dx.doi.org/10.1016/j.ijrefrig.2010.05.001.

[20] H. Kumano, T. Hirata, M. Shirakawa, R. Shouji, Y. Hagiwara, Flow characteristics of ice slurry in narrow tubes, Int. J. Refrig. 33 (8) (2010) 1513-1522, http://dx.doi.org/10.1016/j.ijrefrig.2009.12.002.

[21] A. Delahaye, L. Fournaison, S. Marinhas, M. Martinez, Rheological study of $\mathrm{CO}_{2}$ hydrate slurry in a dynamic loop applied to secondary refrigeration, Chem. Eng. Sci. 63 (13) (2008) 3551-3559, http://dx.doi.org/10.1016/j.ces.2008.04.001.

[22] M. Grozdek, R. Khodabandeh, P. Lundqvist, B. Palm, A. Melinder, Experimental investigation of ice slurry heat transfer in horizontal tube, Int. J. Refrig. 32 (6) (2009) 1310-1322, http://dx.doi.org/10.1016/j.ijrefrig.2009.01.014.

[23] L. Mika, Energy losses of ice slurry in pipe sudden contractions, Exp. Therm. Fluid Sci. 35 (6) (2011) 939-947, http://dx.doi.org/10.1016/j.expthermflusci. 2011.01.013.

[24] V. Fester, B. Mbiya, P. Slatter, Energy losses of non-Newtonian fluids in sudden pipe contractions, Chem. Eng. J. 145 (1) (2008) 57-63, http://dx.doi.org/ 10.1016/j.cej.2008.03.003.

[25] V. Ayel, O. Lottin, H. Peerhossaini, Rheology, flow behaviour and heat transfer of ice slurries a review of the state of the art, Int. J. Refrig. 26 (1) (2003) 95-107, http://dx.doi.org/10.1016/S0140-7007(02)00016-6.

[26] E. Norgaard, T. Sorensen, T. Hansen, M. Kauffeld, Performance of components of ice slurry systems: pumps, plate heat exchangers, and fittings, Int. J. Refrig. 28 (1) (2005) 83-91, http://dx.doi.org/10.1016/j.ijrefrig.2004.07.018.
[27] I. Bellas, S. Tassou, Present and future applications of ice slurries, Int. J. Refrig. 28 (1) (2005) 115-121, http://dx.doi.org/10.1016/j.ijrefrig.2004.07.009.

[28] J. Guilpart, E. Stamatiou, L. Fournaison, The control of ice slurry systems: an overview, Int. J. Refrig. 28 (1) (2005) 98-107, http://dx.doi.org/10.1016/ j.ijrefrig.2004.07.007.

[29] E. Dumont, F. Fayolle, J. Legrand, Electrodiffusional wall shear rate analysis in a scraped surface heat exchanger, AIChE J. 45 (2000) 1138-1148.

[30] P. Pronk, C. Infante Ferreira, G. Witkamp, A dynamic model of Ostwald ripening in ice suspensions, J. Cryst. Growth 275 (1-2) (2005) e1355-e1361, http://dx.doi.org/10.1016/j.jcrysgro.2004.11.173.

[31] B. Stutz, P. Reghem, O. Martinez, Flow of slurries of particle with density close to that of water, in: Fourth International Conference on Multiphase Flow, 2001, pp. 1-12.

[32] H.L. Langhaar, Steady flow in the transition length of a straight tube, J. Appl. Mech. 9 (1942) 55-58.

[33] B. Andreotti, P. Claudin, O. Pouliquen, Measurements of the aeolian sand transport saturation length, Geomorphology 123 (3-4) (2010) 343-348, http://dx.doi.org/10.1016/j.geomorph.2010.08.002.

[34] B. Stutz, P. Reghem, O. Martinez, Friction losses for flow of concentrated slurries, in: Second Workshop on Ice Slurries of IIF/IIR, Paris, no. 1, 2000.

[35] Heat transfer and pressure drop in ice-water slurries, Appl. Therm. Eng. 20 (7) (2000) 671-685

[36] G. Scott, D. Kilgour, The density of random close packing of spheres, J. Phys. D: Appl. Phys. 2 (6) (1969) 863-866, http://dx.doi.org/10.1088/0022-3727/2/6/ 311.

[37] G. Onoda, E. Liniger, Random loose packings of uniform spheres and the dilatancy onset, Phys. Rev. Lett. 64 (22) (1990) 2727-2730.

[38] V. Matousek, Pressure drops and flow patterns in sand-mixture pipes, Exp. Therm. Fluid Sci. 26 (6-7) (2002) 693-702, http://dx.doi.org/10.1016/S08941777(02)00176-0.

[39] S.A. Miedema, Constructing the Shields curve, a new theoretical approach and its applications, in: WODCON XIX, no. September, Beijing, 2010.

[40] D. Edelin, C. Josset, C. Castelain, F. Fayolle, Experimental study of bedforms obtained with floating particles in a pipe flow, J. Fluid Mech. 765 (2015) 252272, http://dx.doi.org/10.1017/jfm.2014.675.

[41] P. Carman, Fluid flow through granular beds, Trans. Inst. Chem. Eng. London 15 (1937) 150-166.

[42] M. Ouriemi, P. Aussillous, M. Medale, Y. Peysson, E. Guazzelli, Determination of the critical Shields number for particle erosion in laminar flow, Phys. Fluids 19 (6) (2007) 061706, http://dx.doi.org/10.1063/1.2747677.

[43] Y. Peysson, M. Ouriemi, M. Medale, P. Aussillous, E. Guazzelli, Threshold for sediment erosion in pipe flow, Int. J. Multiph. Flow 35 (6) (2009) 597-600, http://dx.doi.org/10.1016/j.ijmultiphaseflow.2009.02.007.

[44] M. Ouriemi, P. Aussillous, E. Guazzelli, Sediment dynamics. Part 2. Dune formation in pipe flow, J. Fluid Mech. 636 (2009) 321, http://dx.doi.org/ $10.1017 /$ S0022112009007927.

[45] F. Charru, B. Andreotti, P. Claudin, Sand ripples and dunes, Annu. Rev. Fluid Mech. 45 (1) (2013) 469-493, http://dx.doi.org/10.1146/annurev-fluid011212-140806.

[46] R. Turian, F. Hsu, T. Ma, Estimation of the critical velocity in pipeline flow slurries, Powder Technol. 51 (1987) 35-47.

[47] S.W. Churchill, Friction factor equations spans all fluid-flow ranges, Chem. Eng. J. 91.

[48] V. Matousek, J. Krupicka, Different types of unsteady flow of solids generated in laboratory slurry pipe loop, in: 16 th Int. Conf. on Transport \& Sedimentation of Solid Particles, no. May, 2013.

[49] W. Brostow, Drag reduction in flow: review of applications, mechanism and prediction, J. Ind. Eng. Chem. 14 (2008) 409-416, http://dx.doi.org/10.1016/ j.jiec.2008.07.001. 